\title{
Stress Inoculation Training on Cognitive Emotional Regulation: The Effect on Vulnerable Married Female Adolescents
}

\author{
Hamide Bahaodini ${ }^{1}$, Ghazal Zandekarimi2 ${ }^{*}$ \\ ${ }^{1}$ Guidance and Counseling, Refah University, Tehran, Iran \\ ${ }^{2}$ Department of Psychology, Refah University, Tehran, Iran \\ Email: Hamide.bahaodini@gmail.com, ^ghazalzandi@yahoo.com
}

How to cite this paper: Bahaodini, H. and Zandekarimi, G. (2018) Stress Inoculation Training on Cognitive Emotional Regulation: The Effect on Vulnerable Married Female Adolescents. Journal of Behavioral and Brain Science, 8, 329-338.

https://doi.org/10.4236/jbbs.2018.86021

Received: April 3, 2018

Accepted: May 29, 2018

Published: June 1, 2018

Copyright $\odot 2018$ by authors and Scientific Research Publishing Inc. This work is licensed under the Creative Commons Attribution International License (CC BY 4.0).

http://creativecommons.org/licenses/by/4.0/

(c) (i) Open Access

\begin{abstract}
Married female adolescents are the most vulnerable people that culturally marry with their parents' permission in Iran. This study aimed to investigate the effectiveness of stress inoculation training (SIT) on cognitive emotional regulation (CER) in these women. The method of this research was semi-experiment with pretest and posttest. The statistical society was the married adolescent female students from Qom high schools (80 people). First, they were assessed by cognitive emotional regulation questionnaire to find the subjects under the CER cut of point (55 people). Then 30 participants were randomly selected and were equally divided into experimental and control groups. The entry criteria for samples were female married students between 17 and 19 from low socioeconomic income. The experimental group received 8 sessions of SIT training while there was no intervention for control group. Results from MANCOVA analysis showed that there were significant differences between groups on cognitive emotional regulation in post-test. The data of pretest were the covariate factor. Finding suggests SIT is effective for cognitive emotional regulation in vulnerable married female adolescents. In addition, this training method elevates positive emotional strategies, which correlate with planning, focusing and organizing the emotions. In contrast, except self and the others blaming, negative emotional strategies differed slightly.
\end{abstract}

\section{Keywords}

Cognitive Emotional Regulation, Positive/Negative Emotional Strategies, Stress Inoculation Training 


\section{Introduction}

Family is the smallest unit of the community, which is so important in terms of personal and social effects [1]. In recent decades, many researchers attended to explore the stress outcomes for they play important roles in the quality and stability of couples' relationships and family [2]. Many couples impose plenty of material and spiritual costs because of the difficulty with emotional regulation. Growing number of displeasure, violence, aggression, marital infidelity and..., reveal the importance of regulating emotions. Emotional regulation includes the initiating of new emotional responses and changing current emotional responses that are influenced by regulatory processes [3]. In fact, Emotional regulation strategies might be conscious or unconscious, automated or controlled processes that people involve with them to manage their emotions [4]. Emotional regulation includes two categories of positive and negative strategies. Positive strategies are positive refocusing, focus on planning, positive reappraisal and putting into perspective. Therefore, positive re-focusing on planning contains thoughts about what steps should be taken and how to cope with negative events. Focusing on planning is described as thinking about what steps should be taken and how to cope with negative events. Creating a positive meaning of events in periods of individual growth is named positive reappraisal. Eventually, putting into perspective contains abandoning the seriousness and importance of the event and emphasizing its relativity comparing with other events. Besides, negative strategies contain self-blaming, blaming the others, ruminating, catastrophizing and acceptance. Self-blame represents the thoughts that blame individual experiences. Blaming the others includes the thoughts that a person aims to inculpate the others for existing problems. Rumination is getting involved with feelings and thoughts that are emancipated from negative events. Furthermore, catastrophizing includes an explicit emphasis on the terrible experience and finally, acceptance explains thoughts that a person accepts the experiences and abandons his/her role in problems [5].

All negative strategies result in affective and emotional disorders such as depression and anxiety. Instead, anxiety is uniquely characterized by exaggerated threat and damaged assessment. In addition, depression is specifically identified by negative assessment of self, past, and future events [5]. Disability with CER is a very high risk factor for mental and physical health [6]. Constructive emotional regulation skills help people better deal with challenges that endanger emotional well-being. In fact, emotional regulation could help people manage, control and adjust their emotional responses better [7]. Actually, emotional regulation is a cognitive processing and therefore, cognitive behavioral approaches could help to intervene in this regard. One of these training methods is stress inoculation training (SIT), which is presented by Meichenbaum. This method combines a number of the best elements of behavioral and cognitive therapy. The basic principle of SIT is to pay attention to thinking, feeling, behaving and affecting the others as a prerequisite for changing a behavior [8]. In other words, 
SIT strongly and directly emphasizes on person's thoughts, feelings, perceptions and analysis of daily life [9]. Meichenbaum has developed a three-stage model for SIT: First, conceptualization stage is to establish a cooperative relationship between therapist and patient. Moreover, training is about clarifying the stress, the relationship between stress and coping, and the role of thoughts, actions and emotions that create and maintain stress. In second stage, skill acquisition and rehearsal stage will emerge. At this stage, the therapist develops cognitive and behavioral skills. These methods include new information, planning for escape routes, changing negative selfishness to positive selfishness, behavioral effort for relaxation, decisiveness and self-esteem in order to cope with stressful situations [8]. Finally, application and following through stage is to plan and solve the programs, and reduce stress in order to practice the learnt skills in real life [10].

Studies have shown that SIT reduces marital stress and specially diminishes perceived stress in women [11] \& [12], and finally, reduces anxiety, depression and perceived stress in women [13]. It elevates coping strategies [14], and treats traumatized symptoms of war and torture victims [15]. However, the strengths of the mentioned studies include the research project, using a relatively high number of sample size, using the appropriate statistical test, and the presence of both genders in the research, which empower the mentioned research. Nevertheless, the weaknesses of these studies are quasi-experimental design, sampled by available method and lack of follow-up stage. Specially, there are no evidences on training the SIT to social vulnerable people and married adolescent women who are considered as children according to UNICEF.

The present study was conducted aimed at training SIT to improve CER in vulnerable married female adolescents. It should be noted that a few research has been carried out SIT training in our country. Hence, there is a clear gap between studies in this field. On the one hand, it is not surprising that young couples are considered as at-risk groups despite the given life challenges. Obviously, they drew researchers' attention to the fields of behavioral problems and psychological stress. On the other hand, vulnerable married female adolescents are exposed to all dimensions of pressures such as husbands, parents and society expectations. CER training might be important and useful for this group for they experience high stress among people in our community. CER can help couples in high-pressure situations (references). If couples are engaged with the appropriate level of CER, they can manage the challenges better when they are faced with stressful negotiations. The current study is going to find the answer to the following research question:

Is exposure to SIT effective on positive and negative CER of married female adolescent students?

\section{Methods}

\subsection{Study Population}

The present study method was a semi-experimental study with pretest-posttest 
and control group. The statistical population was the vulnerable married female adolescents that were students at the age of 17 to 19 in high schools of Qom. The reason for choosing this age was that they were among the most challenging period due to their age level (adolscence) and cultural conditions. Furthermore, they did not receive any structural training to enter the common life. In this regard, they are subjected to divorce, to conflict and to sacrifice in corporal and psychological torture. In this research, the randomly sampling method was used; all vulnerable married female students in high schools of Qom were tested (80 women). First, they were assessed by cognitive emotional regulation questionnaire to find the subjects under the CER cut of point (55 persons). Then, 30 of them were randomly selected and were equally assigned into two experimental and control group according to the ethical considerations (e.g. participants' informed consent, security, safety and parental permission for they were under 21 years old). The entry criterion for choosing the subjects were being female, lower than mean score in CER questionnaire, age between 17 and 19 years, the marriage length between 1 to 24 months, and low socioeconomic status (the family income was less than one and a half million Tomans per month). Moreover, the group with lower positive CER scores was placed in the experimental group. The exclusion criteria were being single, and female with less than 1 month of marriage or more than 24 months. Besides, having a marriage under the age of 17 and above 19 was another exit criterion. Finally, those whose family income was more than one and a half million per month were excluded from this study.

\subsection{Instruments}

CER questionnaire has 36 items and involves 9 sub-scales [16]. Positive refocusing, positive reappraisal, putting into perspective, refocusing on planning are positive CER strategies. Self-blame, blaming the others, ruminating, acceptance and catastrophizing are negative CER strategies. Alpha coefficient for the subscales of this questionnaire has been reported in the range of 0.71 to 0.81 . The subscales reliability coefficient of this questionnaire has been reported in the range between 0.48 and 0.61 in the re-test after 14-month interval [16]. In a Persian study, alpha coefficients were obtained between 0.68 and 0.79 [17].

SIT Protocol. It includes 8 sessions of SIT (90 minutes in each session every week), that were presented in Table 1.

\subsection{Procedure}

Participants were interviewed according to the ethical considerations (e.g. participants' informed consent, security, and safety). For the experimental group, 8 sessions of SIT, (90 minutes in each session) were trained and, in contrast, the control group did not receive any training. All data were analyzed with SPSS-24.

\section{Results}

Participants' mean age was 17.67 and the mean income was 920.000 Tomans. 
The mean and standard deviations of the variables discussed in the two groups are as in Table 2.

According to Table 2, experimental group means changed in post-test, while control group means did not specifically change. In addition, after the intervention, the participants of experimental group have shown more improvement in the positive strategies than the negative strategies. The mean and standard deviation of CER sub-scales of the experimental and control groups are presented in the pretest and post-test in Table 2.

The inferential data were analyzed by multivariate covariance analysis test to find the significant differences between groups according to SIT intervention. Notably, all the required assumptions for the covariance analysis were considered. The Kolmogorov-Smirnov normality test was applied to evaluate the

Table 1. Summary of training sessions.

\begin{tabular}{cl}
\hline Sessions & \multicolumn{1}{c}{ Target } \\
\hline 1 & $\begin{array}{l}\text { Familiarity and introduce, describing goals and group rules, the need for such educational } \\
\text { programs, motivating and getting involved }\end{array}$ \\
2 & $\begin{array}{l}\text { Acquaintance with cognitive concepts, features of auto negative thoughts and introducing } \\
\text { cognitive errors }\end{array}$ \\
3 & Relaxation and decline tension training \\
4 & Learn how to encounter with negative thoughts \\
5 & Self-talk guided training and the role of negative self-talk in creating stress \\
6 & The focusing of thought methods and distraction techniques training \\
7 & Problem solving skills training \\
8 & Practicing learned skills and applying these skills in stressful situations \\
\hline
\end{tabular}

Table 2. Descriptive data for cognitive emotional regulation subscales.

\begin{tabular}{ccccccccc}
\hline \multirow{2}{*}{ Variables } & \multicolumn{4}{c}{ Control } & \multicolumn{5}{c}{ Experimental } \\
\cline { 2 - 9 } & \multicolumn{2}{c}{ Pretest } & \multicolumn{2}{c}{ Post-test } & Pretest & Post-test \\
\hline M & SD & M & SD & M & SD & M & SD \\
\hline Positive Refocusing & 9.06 & 1.98 & 9.26 & 1.90 & 11.33 & 2.41 & 9.13 & 1.88 \\
Refocus on Planning & 9.86 & 1.84 & 10.40 & 1.68 & 13.46 & 2.89 & 10.00 & 2.23 \\
Positive Reappraisal & 10.33 & 2.12 & 10.66 & 1.58 & 12.26 & 2.57 & 9.93 & 1.66 \\
Putting into Perspective & 10.46 & 2.35 & 10.60 & 2.47 & 13.80 & 2.14 & 11.00 & 2.80 \\
Positive CER & 49.73 & 5.86 & 51.13 & 5.71 & 47.42 & 5.23 & 61.26 & 6.20 \\
Self-Blame & 15.00 & 1.86 & 15.40 & 1.50 & 11.73 & 2.60 & 15.13 & 2.32 \\
Blame the Others & 15.20 & 2.73 & 15.73 & 3.23 & 10.93 & 3.03 & 15.33 & 2.91 \\
Rumination & 12.86 & 3.06 & 13.00 & 2.39 & 9.73 & 2.34 & 13.46 & 3.18 \\
Catastrophizing & 14.33 & 1.95 & 14.06 & 2.76 & 13.06 & 2.68 & 14.53 & 2.41 \\
Acceptance & 9.73 & 1.43 & 9.66 & 1.67 & 10.40 & 2.72 & 10.20 & 1.65 \\
Negative CER & 58.46 & 4.95 & 58.20 & 4.98 & 57.40 & 6.31 & 45.46 & 5.65 \\
\hline
\end{tabular}


positive CER and negative CER data respectively, which showed the null hypothesis was rejected on the lack of data normality (K-S $=0.19,0.14 ; \mathrm{p}<0.20$, $<0.20)$. Therefore, variables are of normal distribution. The Levene's test applied to evaluate the homogeneity of the positive CER and negative CER variances revealed the null hypothesis was rejected on lack of data homogeneity $\left(\mathrm{F}_{(1,13)}=\right.$ $3.68,4.25 ; \mathrm{p}<0.07,<0.08)$. Results of this test represented the variances were homogeneous. Next, it was necessary to testing residuals' normality. For this end, residual diagram was applied and represented the residuals were of normal distribution. Based on testing of the required assumptions we could use the parametric tests. Therefore, multivariate covariate analysis was applied for equal groups. The results of multivariate covariance analysis are presented in Table 3.

As shown in Table 3, in positive CER, the group observed F is 6.84 and significant ( $\mathrm{p}<0.004$ ), as well as observed $\mathrm{F}$ from the effect of the posttest is 15.41 and significant $(\mathrm{p}<0.001)$. In other words, a significant difference is found between mean scores of pretest and post-test in positive CER. In negative CER, group observed $\mathrm{F}$ is 6.65 and significant $(\mathrm{p}<0.01)$, as well as the observed $\mathrm{F}$ of the posttest is 3.34 and significant $(\mathrm{p}<0.009)$. The effect of CER subscales is given in Table 4.

The results of Table 4 show interventions have not been effective on catastrophizing, rumination and acceptance subscales for negative CER $(\mathrm{p}<0.001)$.

\section{Discussion}

The purpose of the present study was to explore the effectiveness of SIT on CER of vulnerable married female adolescents. Moreover, we aimed to extend the knowledge boundaries of couples' training. The researchers found interesting findings after performing the intervention. The main findings are the following.

First, one of the most important findings is the result of significant increase in CER after intervention; however, we found the remarkable result, which was the effect on the cold cognition, rational system and participants' information processing. According to the findings, participants accepted the common life

Table 3. Multivariate covariance analysis of cognitive emotional regulation.

\begin{tabular}{ccccccc}
\hline Variables & Effect & $\begin{array}{c}\text { Sum of } \\
\text { Squares }\end{array}$ & $\begin{array}{c}\text { Degrees of } \\
\text { Freedom }\end{array}$ & $\begin{array}{c}\text { Mean } \\
\text { Squares }\end{array}$ & F & $\begin{array}{c}\text { Significance } \\
\text { Level }\end{array}$ \\
\hline & Group & 303.05 & 1 & 303.05 & 6.84 & .004 \\
& Post Test & 106.24 & 1 & 106.24 & 15.41 & .001 \\
\hline \multirow{2}{*}{ Positive CER } & Error & 530.88 & 26 & 19.66 & & \\
& Total & 101488 & 30 & & & \\
& Group & 150.97 & 1 & 150.97 & 6.65 & .001 \\
& Post Test & 75.88 & 1 & 75.88 & 3.34 & .009 \\
\hline & Error & 589.80 & 26 & 22.68 & & \\
\hline
\end{tabular}


Table 4. Results of covariance analysis sub-scales of cognitive emotional regulation

\begin{tabular}{ccccccc}
\hline & Variables & $\begin{array}{c}\text { Sum of } \\
\text { Squares }\end{array}$ & Df. & $\begin{array}{c}\text { Mean } \\
\text { Squares }\end{array}$ & F & Sig. \\
\hline \multirow{2}{*}{$\begin{array}{c}\text { Negative } \\
\text { CER }\end{array}$} & Self-Blame & 53.61 & 1 & 53.61 & 14.27 & 0.001 \\
& Rlame the Others & 38.62 & 1 & 38.62 & 9.48 & 0.005 \\
& Catastrophizing & 0.01 & 1 & 0.01 & 0.00 & 0.96 \\
\hline \multirow{2}{*}{$\begin{array}{c}\text { Positive } \\
\text { CER }\end{array}$} & Positive Refocusing & 9.93 & 1 & 39.48 & 9.93 & 0.004 \\
& Refocus on Planning & 18.44 & 1 & 103.98 & 18.44 & 0.001 \\
& Positive Reappraisal & 11.30 & 1 & 46.66 & 11.30 & 0.002 \\
& Putting into Perspective & 15.71 & 1 & 61.34 & 15.71 & 0.001 \\
\hline
\end{tabular}

responsibility after intervention. Obviously, marriage at this age is due to the family culture and it causes double pressure on the adolescent women. Therefore, it results in many problems in their CER. This finding is in line with the Valadkhani, et al. [18] and Aneja et al. findings [13].

Second, according to the research findings, the participants positive CER changed significantly after the intervention. This suggests that they progressed after the intervention in positive CER and benefited more from the positive strategies. The most effective subscales that are affected by the intervention are re-focusing on planning, putting into perspective, positive reappraisal, positive re-focusing respectively. Actually, all positive CER are reinforced by SIT. The most important is the SIT effects on planning, organizing, focusing or in the other words, it effected on meta-cognition which called cold cognition. It seems that immunizing against stress will grow up brains information processing system; nevertheless, its effect on rumination was not significant. Wells believed in rumination as the most important part of negative moods etiology [19]. Our findings are in contrast with this theory and are in line with Lee et al. [14] and Khorsandi, et al. [11].

Third, negative CER turned slightly. Self-blame and blame the others are the subscales that decreased significantly against the other negative subscales. It could be explaining that, when a vulnerable adolescent girl drops into a marriage without real rational support, she blames herself and her parents against the stressful marriage necessity. These negative CERs brings her more stressful against spousal tasks. As a result, she feels inefficient and become more negative in emotional regulation. We cannot change cultural structures immediately. However, it is possible to reinforce adolescent women to control their negative emotions and to upgrade their metacognitions. These will help structural conversion in future for they will be the next mothers. Moreover, there has been no change in the acceptance of participants because catastrophizing and rumination did not improve significantly. It seems these subscales intertwine, and as long as 
the participants do not improve their schema of catastrophizing and rumination, they cannot increase their acceptance.

Finally, it is likely that the participants could find improvement faster in positive strategies for it is related to their moods. While negative emotions are related to their childhood and schemas. Consequently, it would be slowly improved. Therefore, positive CER are associated with superficial mental layers of the human; whereas, negative CER are correlated with deeper mental layers of the brain [20]. Hence, the research question was confirmed and SIT was effective on increasing positive CER and negative CER. Accordingly, the control group did not show significant differences in positive CER and negative CER levels in the post-test. In this research, we attempted to enhance women's CER by teaching SIT cognitive-behavioral methods. The findings indicate the effect of this method on increasing CER and its subscales, which can be explained based on the cognitive and behavioral strategies considered in this study. The auto-negative thoughts, cognitive distortions and the replacement of reasonable thoughts play an important role in CERs. Specially, the participants learned to assess their abilities and talents properly. Based on this correct assessment, they should have reasonable thoughts and expectations to be successful in their marital intra and inter-relationships. These reasonable thoughts and expectations reduce the negative experienced emotions, increase the level of positive strategies and thus increase the mental health. Notably, using behavioral techniques, participants could show physical signs of emotions and the sense of increased control. This finding is congruent with the research findings of Khorsandi, et al. [11], Kraaji \& Garnefski [5], and Lee et al. [14].

The present study has several strengths. It also has several practical implications. First, since there are a few research in this field, the results of this study can be indirectly consistent with studies indicating the effectiveness of SIT treatment on improving the psychological health of vulnerable married women. Second, the results of this study can be consistent with a small number of studies that show the effectiveness of cognitive-behavioral treatments on improving the psychological problems of married female adolescents. Both in the literature review in Iran and overseas, there are few research which examine the effectiveness of SIT on married female adolescents between 17 - 19. In this intervention, the concept of adolescent women stressful life has attracted the attention of women community in Iran. Finally, the present study showed that if women become skilled, ignoring the effect size, CER will improve and possibly, we could see cultural conversion in future.

Regarding the limitations of this work, the present study has several implications. First, one of the limitations is that it was done only in the female married adolescents so its generalization to the single adolescents needs further research in this regard. Second, low socio-economic level was considered in this study and therefore, other socio-economic levels were not considered. Finally, and unfortunately, it has not been possible to compare the results obtained in this 
study with the results of previous studies. Because, the participants did not have any training about life and marital skills, and they are considered as children in accordance with UNICEF. In addition, the lack of follow-up stage due to administrative constraints prevented the possibility of examining the effectiveness of the intervention in the long term. Therefore, supplementary studies on gender, age and socio-economic levels are recommended in the field of SIT therapies with follow-up stages. Moreover, charities and women's rights activists have to establish community-based organizations that can take effective action to support the empowerment of the psychological skills of girls and women.

\section{Conclusion}

Generally, SIT training course, relaxation skills, identifying auto-negative thoughts and cognitive distortions, replacing reasonable thoughts, learning problem solving methods, and other SIT skills control stress in vulnerable married female adolescents. These skills could reinforce the positive CER and consequently, decline the negative CER. SIT training could reduce self and the others blaming and increase planning, focusing, and organizing the emotions of vulnerable married female adolescents.

\section{Acknowledgements}

Funding for this work was provided by Refah University. The authors thank the participating vulnerable female married students who assisted in interpretation of the results. The authors declare that they have no competing or potential conflicts of interest. All ethical considerations (e.g. participants' informed consent, security, safety and parental permission for they were under 21 years old) were regarded. The third author takes full responsibility for the integrity of the data and the accuracy of the data analyses.

\section{References}

[1] Birjandi, S.M. (2015) Theories and Techniques of Family Therapy 2 Revised. Roshd, Tehran.

[2] Randall, A.K. and Bodenmann, G. (2009) The Role of Stress on Close Relationships and Marital Satisfaction. Clinical Psychology Review, 29, 105-115.

https://doi.org/10.1016/j.cpr.2008.10.004

[3] Kevin, N., Ochsner, K.N. and Gross, J.J. (2005) The Cognitive Control of Emotion. TRENDS in Cognitive Sciences, 9, 242-249. https://doi.org/10.1016/j.tics.2005.03.010

[4] Hagan, C. (2015) Emotion Regulation and Adherence to Display Rules after Experiencing Childhood Trauma. MA Research, Duquesne University, Pittsburgh.

[5] Kraaji, V. and Garnefski, N. (2015) Cognitive, Behavioral and Goal Adjustment Coping and Depressive Symptoms in Young People with Diabetes: A Search for Intervention Targets for Coping Skills Training. Journal of Clinical Psychology \& Medical Settings, 22, 45-53. https://doi.org/10.1007/s10880-015-9417-8

[6] Zhang, H., Wang, Z., You, X., Lu, W. and Luo, Y. (2015) Association between Nar- 
cissism and Emotional Regulation Difficulties: Respiratory Sinus Arrhythmia Reactivity as a Moderator. Journal of Biological Psychology, 110, 1-11.

https://doi.org/10.1016/j.biopsycho.2015.06.014

[7] Eftekhari, A., Zoellner, L.A. and Vigil, S.A. (2009) Patterns of Emotion Regulation and Psychopathology. Anxiety Stress Coping, 22, 571-586.

https://doi.org/10.1080/10615800802179860

[8] Corey, G. (2012). Theory and practice of counseling and psychotherapy $9^{\text {th }}$ Edition. US: Cengage Learning

[9] Lancaster, C.L., Teeters, J.B., Gros, D.F. and Back, S.E. (2016) Posttraumatic Stress Disorder: Overview of Evidence-Based Assessment and Treatment. Journal of Clinical Medicine, 5, 105. https://doi.org/10.3390/jcm5110105

[10] Seligman, L. and Reichenberg, L.W. (2009) Theories of Counseling and Psychotherapy: Systems, Strategies, and Skills. 3rd Edition, Pearson, New Jersey.

[11] Khorsandi, M., Vakilian, K. and Salehi, B. (2016) The Effects of Stress Inoculation Training on Perceived Stress in Pregnant Women. Journal of Health Psychology, 21, 2977-2982. https://doi.org/10.1177/1359105315589800

[12] Serino, S., Triberti, S., Villani, D., Cipresso, P., Gaggioli, A. and Riva, G. (2014) Toward a Validation of Cyber-Interventions for Stress Disorders Based on Stress Inoculation Training: A Systematic Review. Virtual Reality, 18, 73-87. https://doi.org/10.1007/s10055-013-0237-6

[13] Aneja, J., Chavan, B. S., Huria, A., Goel, P., Kohli, N. and Chhabra, P. (2017) Perceived Stress and Its Psychological Correlates in Pregnant Women: An Indian Study. International Journal of Culture and Mental Health, 33, 1-12. https://doi.org/10.1080/17542863.2017.1364284

[14] Lee, A.G., Buckmaster, Ch.L., Yi, E., Schatzberg, A.F. and Lyons, D.M. (2014) Coping and Glucocorticoid Receptor Regulation by Stress Inoculation. Psycho-NeuroEndocrinology, 49, 272-279. https://doi.org/10.1016/j.psyneuen.2014.07.020

[15] Hensel-Dittmann, D., Schauer, M., Ruf, M., Catani, C., Odenwald, M., Elbert, T. and Neuner, F. (2011) Treatment of Traumatized Victims of War and Torture: A Randomized Controlled Comparison of Narrative Exposure Therapy and Stress Inoculation Training. Psychotherapy and Psychosomatics, 80, 345-352. https://doi.org/10.1159/000327253

[16] Garnefski, N., Kraaij, V. and Spinhoven, P. (2002) Manual for the Use of the Cognitive Emotion Regulation Questionnaire. DATEC, Kalamazoo.

[17] Pyvastegar, M. and Heidariabdy, A. (2008) The Comparison of Relationship Cognitive Emotion Regulation Strategies with Depressive Symptoms in Clinical and Non-Clinical Adolescent. Journal of Applied Psychology, 2, 549-563.

[18] Valadkhani, M., Mahmoudpour, A., Farahbakhsh, K. and Salimi Bajestani, H. (2017) The Effects of Partner-Selection Patterns, Marriage Age, and Age Differences between Spouses on Marital Quality of Married Women in Tehran. Quarterly Journal of Clinical Psychology, 7, 173-190.

[19] Wells, A. (2000) Emotional Disorders and Metacognition: Innovate Cognitive Therapy. Wiley, Chichester.

[20] Young, J.E., Klosko, J.S. and Weishaar, M.E. (2003) Schema Therapy: A Practitioners Guide. The Guilford Press, New York. 